\title{
Impact of turnaround time on outcome with point-of-care testing for respiratory viruses: a post hoc analysis from a randomised controlled trial
}

\begin{abstract}
To the Editor:
Respiratory viruses are detected in around $40-50 \%$ of adults hospitalised with acute respiratory illness (ARI) $[1,2]$. Routine laboratory PCR testing for respiratory viruses generally takes several hours to several days to generate results for clinicians and so cannot be used to inform decision making in real time. Decisions about hospitalisation, antibiotics, antivirals and side room isolation therefore need to be made presumptively and reviewed when results are available. Newer rapid molecular test platforms are accurate, easy to use, and generate a result in $1 \mathrm{~h}$ or less, making them potentially deployable for point-of-care testing (POCT) in clinical areas [3]. Recently, we reported on a large pragmatic randomised controlled trial (ResPOC) [4] which evaluated the impact of POCT (using the FilmArray Respiratory Panel (BioFire Diagnostics, Salt Lake City, UT, USA) which tests for a comprehensive range of viruses) in adults presenting to hospital with ARI. The study showed that POCT was associated with reductions in hospital length of stay overall and reductions in antibiotics use in patients with exacerbation of airways disease. Although this evidence would suggest that rapid molecular testing needs to be performed within clinical areas for these improved clinical outcomes, it has been suggested that rapid molecular test platforms used within centralised laboratories might also be associated with these clinical benefits, although the turnaround times (TATs) are likely to be much longer. In this follow-on study we evaluate the impact of POCT TAT on clinical outcomes with a view to determining how rapid molecular testing for respiratory viruses should be best implemented in clinical practice.
\end{abstract}

The study design, inclusion and exclusion criteria, outcome measures and baseline population characteristics of the ResPOC study have been previously described in the original report of this trial [4]. The study was approved by the North West-Preston Regional Ethics Committee (NW/14/1467). The protocol is published and freely available [5].

A post hoc analysis was performed to explore the impact of the POCT TAT on clinical outcomes having previously shown significant differences between the POCT group and the control group for overall length of stay and antibiotic use. TAT is defined as the time from a patient being recruited to the results being communicated to clinicians. As our previous study demonstrated that the improved outcomes seen with POCT occur only in patients testing positive for viruses (with those testing negative having similar outcomes to control patients) we restricted our analysis to those patients testing positive for viruses by POCT. We examined the association between POCT TAT, length of stay and antibiotic use and assessed the effect of a TAT of less than or greater than $1.6 \mathrm{~h}$ (the median). Statistical analyses were performed using Prism version 7.0 (Graphpad software, La Jolla, CA, USA) and Stata version 13.1 (StataCorp, College Station, TX, USA). Correlation was assessed using Spearman's $r_{\mathrm{s}}$. We compared length of stay and antibiotic use between groups using median differences and the Mann-Whitney U-test and differences in proportions using the Chi-squared test or Fisher's exact test, as appropriate. Receiver operating characteristic (ROC) curves were generated to determine the optimal cut-off for TAT. We performed a subgroup analysis of patients who tested positive for influenza A or B and for patients who tested positive for rhinovirus.

@ERSpublications

As very rapid turnaround times lead to better outcomes, virus diagnostics should be performed at the point-of-care http://ow.ly/eD6p30koZGg

Cite this article as: Brendish NJ, Malachira AK, Beard KR, et al. Impact of turnaround time on outcome with point-of-care testing for respiratory viruses: a post hoc analysis from a randomised controlled trial. Eur Respir J 2018; 52: 1800555 [https://doi.org/10.1183/13993003.00555-2018]. 
Of the 720 patients recruited in the parent randomised controlled trial, 360 allocated to the intervention (POCT) group and 354 allocated to the control (routine clinical care) group were included in the original analysis. TATs for POCT results varied from 1.1 to $6.4 \mathrm{~h}$ with a median (interquartile range (IQR)) of $1.6 \mathrm{~h}$ (1.3 to 3.1) compared to a median of $29.8 \mathrm{~h}$ (24.7 to 45.8$)$ for laboratory PCR in the control group. Of the 360 patients tested for respiratory viruses by POCT, 153 (43\%) were positive. Human rhinovirus (55 out of 153 patients (36\%)) and Influenza A and B (53 out of 153 patients (35\%)) were the most commonly detected viruses.

For patients testing positive for viruses by POCT ( $\mathrm{n}=153)$, the median (IQR) for TAT was $1.6 \mathrm{~h}$ (1.3 to 3.0). Sixteen out of 153 patients (10\%) were discharged directly from the emergency department (ED). For patients admitted to hospital $(\mathrm{n}=137)$, TAT was positively correlated with length of hospital stay $\left(\mathrm{r}_{\mathrm{s}} 0.24\right.$ ( $95 \%$ CI 0.07 to 0.39 ); $\mathrm{p}=0.0051)$ and duration of antibiotics $\left(\mathrm{r}_{\mathrm{s}} 0.22\right.$ (95\% CI 0.05 to 0.38 ); $\mathrm{p}=0.0096$ ).

There was no difference in the proportions discharged directly from the ED with a TAT of $\leqslant 1.6 \mathrm{~h}$ versus a TAT of $>1.6 \mathrm{~h}$ (eight out of 77 patients (10\%) versus eight out of 76 patients (11\%); odds ratio (OR) 1.0 (95\% CI 0.3 to 2.8$) ; \mathrm{p}=0.99)$. For those admitted $(\mathrm{n}=137)$, the median (IQR) length of stay was 2.3 days (1.0 to 4.0 ) for TATs of $\leqslant 1.6 \mathrm{~h}$ versus 5.1 days (2.4 to 8.3) for TATs of $>1.6 \mathrm{~h}$ (a difference of 2.8 days (95\% CI 1.0 to 3.5$) ; \mathrm{p}<0.0001$ ). This difference in length of stay was due to a higher proportion of patients with a TAT of $\leqslant 1.6 \mathrm{~h}$ being discharged within $24 \mathrm{~h}$ of admission (18 out of 69 patients (26\%) versus nine out of 68 patients (13\%); OR 2.3 (95\% CI 1.0 to 5.4); $\mathrm{p}=0.058$; number needed to test $=8$ ) or within $48 \mathrm{~h}$ of admission (34 out of 69 patients (49\%) versus 15 out of 68 patients (22\%); OR 3.4 (95\% CI 1.6 to 7.0 ); $\mathrm{p}=0.0012$; number needed to test $=4$ ).

A smaller proportion of patients with a TAT of $\leqslant 1.6 \mathrm{~h}$ versus $>1.6 \mathrm{~h}$ were treated with antibiotics (55 out of 69 patients ( $80 \%$ ) versus 63 out of 68 patients (93\%); OR 0.3 (95\% CI 0.1 to 0.9 ); $\mathrm{p}=0.029$; number needed to test $=8$ ). The median (IQR) duration of antibiotics was 2.9 days $(0.1$ to 6.9$)$ for a TAT of $\leqslant 1.6 \mathrm{~h}$ versus 6.5 days (2.4 to 8.5 ) for a TAT of $>1.6 \mathrm{~h}$ (a difference of 3.6 days (95\% CI 0.1 to $4.2 ; \mathrm{p}=0.0097$ ). This was due to a higher proportion of patients with a TAT $\leqslant 1.6 \mathrm{~h}$ receiving $<24 \mathrm{~h}$ of antibiotics (29 out of 69 patients (42\%) versus 16 out of 68 patients (23\%); OR 2.3 (95\% CI 1.1 to 5.0); p=0.021; number needed to test=5) and $<48 \mathrm{~h}$ of antibiotics (32 out of 69 patients (46\%) versus 17 out of 68 patients (25\%); OR 2.6 ( $95 \%$ CI 1.3 to 5.4); $\mathrm{p}=0.012$; number needed to test $=5$ ) compared to those with a TAT of $>1.6 \mathrm{~h}$ for POCT.

ROC curve analysis showed that a TAT cut-off of $<1.6 \mathrm{~h}$ had optimal sensitivity and specificity for association with early discharge (48\% (95\% CI 32 to 56) and 77\% (95\% CI 65 to 87), respectively; area under the curve (AUC) 0.68; $\mathrm{p}=0.0002$ ) and early discontinuation of antibiotics ( $45 \%$ (95\% CI 33 to 57 ) and 74\% (95\% CI 23 to 84), respectively; AUC 0.61; $\mathrm{p}=0.021$ ).

Forty-nine out of 53 influenza positive patients (92\%) were admitted to hospital, as were (excluding viral co-infections) 46 out of 51 rhinovirus positive patients (96\%). Subgroup analysis for hospitalised influenza and rhinovirus positive patients showed that a rapid TAT $(<1.6 \mathrm{~h})$ was associated with shorter length of stay and shorter antibiotic duration for influenza positive patients but not for rhinovirus positive patients, although the numbers in the individual groups were small. Results for the main cohort and the subgroups of influenza patients and rhinovirus positive patients are shown in table 1 .

This study shows that, even with the rapid TATs for results seen with molecular POCT compared to centralised laboratory PCR testing, TAT remains an important determinant of clinical decision making for respiratory virus testing. In adults with ARI, very rapid TATs are associated with higher rates of early discharge and early discontinuation of antibiotics compared to longer TATs. This suggests that there is a brief and early "window period" for the results of respiratory virus testing to alter patient management after admission to hospital. Although the TAT of laboratory PCR testing is variable across different institutions and may be as short as several hours in some centres, a very short TAT of under $2 \mathrm{~h}$ is unlikely to be achievable within centralised laboratories and so rapid molecular viral diagnostics should be performed in clinical areas at the point-of-care in order to realise these clinical benefits.

Although this study is a post hoc analysis, its strengths include the randomised nature of the parent study, the large cohort of patients studied and its pragmatic nature. In addition, our findings are consistent with observational studies using rapid molecular diagnostics for respiratory viruses which show improvements in clinical outcome dependent on short TATs [6,7]. Although the changes seen are likely to be generalisable to other centres, we cannot rule out that they are dependent on the processes of care in UK hospitals. Although there was no measured increase in adverse events in the group associated with premature discharge and reduced antibiotic use, we cannot exclude a subsequent increase in primary care visits post-discharge, as this data was not available to us.

The cost effectiveness of a routine molecular POCT strategy for respiratory viruses in hospitalised adults is currently unknown. As length of hospital stay is the key determinant of cost for patients hospitalised with 
TABLE 1 Diagnosis and outcomes by diagnostic group for patients testing positive for viruses $(\mathrm{n}=153$ ) with respect to point-of-care testing (POCT) turnaround time (TAT)

Total subjects

\begin{tabular}{|c|c|c|c|c|c|c|}
\hline & Total subjects & POC & TAT & Difference $(95 \% \mathrm{CI})$ & OR $(95 \% \mathrm{CI})$ & p-value \\
\hline & & $\leqslant 1.6 \mathrm{~h}$ & $>1.6 \mathrm{~h}$ & & & \\
\hline Diagnosis (all patients) & & & & & & \\
\hline Subjects & 153 & 77 & 76 & & & \\
\hline Exacerbation asthma/COPD & 153 & $32 / 77(42)$ & $30 / 76$ (39) & & $1.1(0.6$ to 2.1$)$ & 0.87 \\
\hline CAP & 153 & 13/77 (17) & $17 / 76(22)$ & & $0.7(0.3$ to 1.6$)$ & 0.42 \\
\hline ILI/NPLRTI & 153 & 24/77 (31) & $22 / 76(29)$ & & $1.1(0.6$ to 2.2$)$ & 0.86 \\
\hline Other & 153 & $8 / 77(10)$ & $7 / 76(9)$ & & $1.1(0.4$ to 3.0$)$ & 0.99 \\
\hline Severity (all patients) & & & & & & \\
\hline Pulse rate beats $\cdot \mathrm{min}^{-1}$ & 153 & 105 (90 to 120$)$ & 100 (88 to 110$)$ & $-5.5(-12$ to 0$)$ & & 0.055 \\
\hline Respiratory rate breaths $\cdot \min ^{-1}$ & 153 & 25 (20 to 28) & $20(18$ to 26$)$ & $-5(-5$ to -1$)$ & & 0.0012 \\
\hline Systolic BP $\mathrm{mmHg}$ & 153 & 134 (118 to 153$)$ & $132(117$ to 150$)$ & $-2(-9$ to 6$)$ & & 0.74 \\
\hline Saturations $\%$ & 153 & 96 (93 to 98) & 96 (93 to 98) & $0(-1$ to 1$)$ & & 0.76 \\
\hline $\mathrm{CRP} \mathrm{mg} \cdot \mathrm{L}^{-1}$ & 153 & 37 (16 to 93 ) & $61(12$ to 129$)$ & $24(-10$ to 24$)$ & & 0.61 \\
\hline WCC $\times 10^{9}$ per $\mathrm{L}$ & 153 & 10.8 (7.6 to 15.2$)$ & $10.2(7.9$ to 13.2$)$ & $-0.6(-2$ to 0.9$)$ & & 0.47 \\
\hline Outcomes & & & & & & \\
\hline All patients & & & & & & \\
\hline Subjects & 153 & 77 & 76 & & & \\
\hline Discharged from ED & 153 & $8 / 77(10)$ & $8 / 76(11)$ & & $1.0(0.3$ to 2.8$)$ & 0.99 \\
\hline Admitted & 153 & 69/77 (90) & 68/76 (89) & & $1.0(0.4$ to 2.9$)$ & 0.99 \\
\hline Length of hospital stay days & 137 & $2.3(1.0$ to 4.0$)$ & 5.1 (2.4 to 8.3$)$ & 2.8 (1.0 to 3.5$)$ & & $<0.0001$ \\
\hline Discharged within $24 \mathrm{~h}$ & 137 & $18 / 69(26)$ & $9 / 68(13)$ & & $2.3(1.0$ to 5.4$)$ & 0.058 \\
\hline Discharged within $48 \mathrm{~h}$ & 137 & $34 / 69$ (49) & $15 / 68(22)$ & & $3.4(1.6$ to 7.0$)$ & 0.0012 \\
\hline Treated with antibiotics & 137 & $55 / 69(80)$ & 63/68 (93) & & $0.3(0.1$ to 0.9$)$ & 0.029 \\
\hline Duration of antibiotics days & 137 & $2.9(0.1$ to 6.9$)$ & $6.5(2.4$ to 8.5$)$ & $3.6(0.1$ to 4.2$)$ & & 0.0097 \\
\hline Treated with $<24 \mathrm{~h}$ antibiotics & 137 & $29 / 69(42)$ & $16 / 68(23)$ & & $2.3(1.1$ to 5.0$)$ & 0.021 \\
\hline Treated with $<48 \mathrm{~h}$ antibiotics & 137 & $32 / 69(46)$ & $17 / 68(25)$ & & $2.6(1.3$ to 5.4$)$ & 0.012 \\
\hline Influenza positive only & & & & & & \\
\hline Subjects & 49 & 23 & 26 & & & \\
\hline Length of hospital stay days & 49 & 2.0 (0.9 to 3.7$)$ & $5.1(1.8$ to 7.1$)$ & $3.1(0.3$ to 4.2$)$ & & 0.023 \\
\hline Discharged within $24 \mathrm{~h}$ & 49 & $7 / 23(30)$ & $4 / 26(15)$ & & $2.4(0.6$ to 8.2$)$ & 0.31 \\
\hline Discharged within $48 \mathrm{~h}$ & 49 & $12 / 23(52)$ & $7 / 26(27)$ & & $3.0(0.9$ to 10.0$)$ & 0.086 \\
\hline Treated with antibiotics & 49 & $18 / 23(78)$ & $25 / 26(96)$ & & $0.1(0.1$ to 1.3$)$ & 0.086 \\
\hline Duration of antibiotics days & 49 & $1.1(0.1$ to 6.9$)$ & 7.0 (3.8 to 8.9$)$ & $5.9(0.3$ to 6.1$)$ & & 0.0048 \\
\hline Treated with $<24 \mathrm{~h}$ antibiotics & 49 & $11 / 23(48)$ & $6 / 26(23)$ & & 3.1 (0.8 to 9.5 ) & 0.082 \\
\hline Treated with $<48 \mathrm{~h}$ antibiotics & 49 & $12 / 23(52)$ & $6 / 26(23)$ & & $3.6(1.0$ to 11.2$)$ & 0.043 \\
\hline Treated with NAls & 49 & $23 / 23(100)$ & $24 / 26(92)$ & & 4.4 (0.2 to 97 ) & 0.34 \\
\hline Rhinovirus positive only & & & & & & \\
\hline Subjects & 46 & 29 & 17 & & & \\
\hline Length of hospital stay days & 46 & $2.4(1.0$ to 4.5$)$ & $2.6(1.0$ to 8.5$)$ & $0.2(-1.0$ to 2.3$)$ & & 0.44 \\
\hline Discharged within $24 \mathrm{~h}$ & 46 & $7 / 29(24)$ & $4 / 17(24)$ & & $1.0(0.3$ to 3.6$)$ & 1.0 \\
\hline Discharged within $48 \mathrm{~h}$ & 46 & $13 / 29(45)$ & $5 / 17(29)$ & & $2.0(0.6$ to 6.6$)$ & 0.36 \\
\hline Treated with antibiotics & 46 & 23/29 (79) & 15/17 (88) & & $0.5(0.1$ to 2.6$)$ & 0.69 \\
\hline Duration of antibiotics days & 46 & $6.0(0.1$ to 7.5$)$ & $6.4(3.1$ to 8.6$)$ & $0.4(-0.7$ to 5.4$)$ & & 0.44 \\
\hline Treated with $<24 \mathrm{~h}$ antibiotics & 46 & $11 / 29(38)$ & $4 / 17(22)$ & & $2.1(0.6$ to 7.0$)$ & 0.34 \\
\hline Treated with $<48 \mathrm{~h}$ antibiotics & 46 & $12 / 29(41)$ & $4 / 17(24)$ & & $2.2(0.6$ to 7.6$)$ & 0.34 \\
\hline Safety (all patients) ${ }^{\#}$ & & & & & & \\
\hline ICU admission & 137 & $1 / 77(1)$ & $3 / 76(4)$ & & $0.3(0.1$ to 2.2$)$ & 0.37 \\
\hline Death & 153 & $0 / 77(0)$ & $0 / 76(0)$ & & & 1.0 \\
\hline Representation to ED & 153 & $6 / 77(8)$ & $6 / 76(8)$ & & $0.5(0.2$ to 1.5$)$ & 0.21 \\
\hline Readmission & 138 & $4 / 77(6)$ & $5 / 76(7)$ & & $0.8(0.2$ to 2.7$)$ & 0.72 \\
\hline
\end{tabular}

Difference $(95 \% \mathrm{CI})$

OR $(95 \% \mathrm{Cl}) \quad \mathrm{p}$-value

Data is presented as $n, n / n(\%)$ or mean (interquartile range) unless otherwise stated. $p$-Values in bold indicate statistical significance, as defined by $p \leqslant 0.05$. COPD: chronic obstructive pulmonary disease; CAP: community-acquired pneumonia; ILI: influenza-like illness; NPLRTI: non-pneumonic lower respiratory tract infection; BP: blood pressure; CRP: C-reactive protein; WCC: white cell count; NAI: neuraminidase inhibitor; ED: emergency department; ICU: intensive care unit; OR: odds ratio. ${ }^{\#}$ : measured for 30 days post enrolment.

ARI, the increase in premature discharge with POCT strongly suggests that even a modestly more expensive diagnostic strategy is likely to be a cost saving compared to routine clinical care. It is currently uncertain as to how molecular POCT for respiratory viruses could be implemented within the NHS and 
other health systems. Potential models include training clinical staff to perform the testing or the development of dedicated point-of-care testing laboratories within or close to acute areas.

In summary POCT with a TAT of $<1.6 \mathrm{~h}$ was associated with higher rates of early hospital discharge and early discontinuation of antibiotics compared to longer TATs. As these very rapid TATs are unlikely to be achievable with centralised laboratory testing, viral diagnostics should be performed at the point of care and models for the implementation of this strategy need to be explored.

Nathan J. Brendish $\oplus^{1,2}$, Ahalya K. Malachira ${ }^{3}$, Kate R. Beard ${ }^{1}$, Sean Ewings ${ }^{4}$ and Tristan W. Clark ${ }^{1,3,5}$

${ }^{1}$ Academic Unit of Clinical and Experimental Sciences, University of Southampton, Southampton, UK. ${ }^{2}$ NIHR Southampton Clinical Research Facility, University Hospital Southampton NHS Foundation Trust, Southampton, UK. ${ }^{3}$ Dept of Infection, University Hospital Southampton NHS Foundation Trust, Southampton, UK. ${ }^{4}$ Statistical Sciences Research Institute, University of Southampton, Southampton, UK. ${ }^{5}$ NIHR Southampton Biomedical Research Centre, University Hospital Southampton NHS Foundation Trust, Southampton, UK.

Correspondence: Tristan W. Clark, LF101, South Academic Block, Southampton General Hospital, Southampton, SO16 6YD, UK. E-mail: T.W.Clark@soton.ac.uk

Received: Jan 312018 | Accepted after revision: May 302018

This study is registered with International Standard Randomised Controlled Trial Number (ISRCTN) 90211642.

Acknowledgements: We thank all of the patients and clinical staff at the Southampton General Hospital, including clinicians, nurses and laboratory technicians. We thank the directors, research nurses, data managers, clinical trials assistants and laboratory staff at the NIHR Southampton Clinical Research Facility and the NIHR Southampton Biomedical Research Centre. We thank the staff at the R\&D Department, University Hospital Southampton NHS Foundation Trust and the NIHR Clinical Research Network, Wessex for their support throughout the trial.

Conflict of interest: N.J. Brendish has been an investigator and recruited patients into clinical trials of antivirals sponsored by Gilead and Janssen. T.W. Clark reports personal fees for consultancy work from Roche, speaker fees, travel expenses, equipment and consumables for purposes of research from BioFire LLC, outside the submitted work. He has been a principal investigator or chief investigator for industry sponsored trials of antivirals (Gilead, Janssen) and has been an investigator and recruited patients into clinical trials sponsored by Novartis, Baxter and GSK.

Support statement: The parent trial was funded by the University of Southampton in the form of an internal grant. This report is independent research supported by the National Institute for Health Research (NIHR) via a Post-Doctoral Fellowship for T.W. Clark (PDF 2016-09-061). The corresponding author had full access to all of the data and the final responsibility to submit for publication. The views expressed are those of the author(s) and not necessarily those of the NHS, the National Institute for Health Research (NIHR) or the Department of Health. The manufacturers of the molecular test platform (Biofire, Salt Lake City, UT, USA) had no role in the conception or design of the study, in data analysis, or in manuscript preparation. Funding information for this article has been deposited with the Crossref Funder Registry.

\section{References}

1 Clark TW, Medina MJ, Batham S, et al. Adults hospitalised with acute respiratory illness rarely have detectable bacteria in the absence of COPD or pneumonia; viral infection predominates in a large prospective UK sample. J Infect 2014; 69: 507-515.

2 Falsey AR, Becker KL, Swinburne AJ, et al. Bacterial complications of respiratory tract viral illness: a comprehensive evaluation. J Infect Dis 2013; 208: 432-441.

3 Brendish NJ, Schiff HF, Clark TW. Point-of-care testing for respiratory viruses in adults: the current landscape and future potential. J Infect 2015; 71: 501-510.

4 Brendish N, Malachira A, Armstrong L, et al. Routine molecular point-of-care testing for respiratory viruses in adults presenting to hospital with acute respiratory illness (ResPOC): a pragmatic, open-label, randomised controlled trial. Lancet Respir Med 2017; 5: 401-411.

5 Brendish NJ, Malachira AK, Clark TW. Molecular point-of-care testing for respiratory viruses versus routine clinical care in adults with acute respiratory illness presenting to secondary care: a pragmatic randomised controlled trial protocol (ResPOC). BMC Infect Dis 2017; 17: 128.

6 Chu HY, Englund JA, Huang D, et al. Impact of rapid influenza PCR testing on hospitalization and antiviral use: a retrospective cohort study. J Med Virol 2015; 87: 2021-2026.

7 Rogers BB, Shankar P, Jerris RC, et al. Impact of a rapid respiratory panel test on patient outcomes. Arch Pathol Lab Med 2015; 139: 636e41. 